Photoconductive Detectors with Fast Temporal Response for Laser Produced Plasma Experiments.

MJ May, C Halvorson, T Perry, F Weber, P Young, C Silbernagel

May 7, 2008

High Temperatuer Plasma Diagnostics Conference Albuquerque, NM, United States

May 11, 2008 through May 15, 2008 
This document was prepared as an account of work sponsored by an agency of the United States government. Neither the United States government nor Lawrence Livermore National Security, LLC, nor any of their employees makes any warranty, expressed or implied, or assumes any legal liability or responsibility for the accuracy, completeness, or usefulness of any information, apparatus, product, or process disclosed, or represents that its use would not infringe privately owned rights. Reference herein to any specific commercial product, process, or service by trade name, trademark, manufacturer, or otherwise does not necessarily constitute or imply its endorsement, recommendation, or favoring by the United States government or Lawrence Livermore National Security, LLC. The views and opinions of authors expressed herein do not necessarily state or reflect those of the United States government or Lawrence Livermore National Security, LLC, and shall not be used for advertising or product endorsement purposes. 


\title{
Photoconductive Detectors with Fast Temporal Response for Laser Produced Plasma Experiments.
}

\author{
MJ May, C. Halvorson, T. Perry, F. Weber, P. Young \\ Lawrence Livermore National Laboratory, 7000 East Ave. Livermore, CA 94550 \\ C. Silbernagel
}

National Security Technologies LLC, P.O. Box 2710, Livermore, CA 94550

\begin{abstract}
Processes during laser plasma experiments typically have time scales that are less than $100 \mathrm{ps}$. The measurement of these processes requires X-ray detectors with fast temporal resolution. We have measured the temporal responses and linearity of several different Xray sensitive Photoconductive Detectors (PCDs). The active elements of the detectors investigated include both diamond (natural and synthetic) and GaAs crystals. The typical time responses of the GaAs PCDs are approximately $60 \mathrm{ps}$, respectively. Some characterizations using X-ray light from a synchrotron light source are presented.
\end{abstract}

\section{Key Words: HTPD2008, High Bandwidth Detectors}




\section{Introduction:}

High energy density experiments at high energy density (HED) laser plasma and pulsed power facilities create transient plasmas with durations of 1 to $10+\mathrm{ns}$. Experimenters wish to measure the time history of the emission from the ultraviolet, $\mathrm{X}$ ray, gamma ray, etc. from the source with high bandwidths or with time resolutions of 0.1 ns or better ${ }^{12}$. To accomplish these measurements requires not only fast data transmission systems but also high bandwidth detectors that are sensitive to the radiation of interest.

For the X-ray spectral band typical detectors are the X-ray diode (XRD) or microchannel plate detector (MCP). XRDs can have speed around 150 ps or better and have been used on laser plasma experiments and $\mathrm{Z}$ pulsed power experiments at Sandia National $\mathrm{Labs}^{3}$. One detector that can potentially can record signals with $10 \mathrm{ps}$ time resolution is the photoconductive detector (PCD). There are several types of commercially available PCDs. They can be made from either diamond or GaAs. NuTrek has recently produced GaAs PCDs that promise sub picosecond response ${ }^{4}$. Diamond PCDs have been successfully used for X-ray measurements in laser produced plasma experiments at the OMEGA Laser facility at the Lab for Laser Energetics ${ }^{5}$.

\section{Photoconductive Detectors:}

In an (XRD), an incident photon produces an electron in the cathode material through the photoelectric effect. The liberated electrons in the detector become the detected current. The signal production mechanism in a PCD is much different from that of an XRD. The PCD works through a change in conductivity. When particles or light of energy greater than the band gap are absorbed in the material, free electrons and holes are created. The excited free carriers have high mobility and this increases the conductivity. The difference in the conductivity with an applied radiation source is much larger than without the source since the number of thermally generated carriers is negligible.

With the application of a bias voltage, V, the current flowing through a PCD can be expressed as:

$$
I=A \frac{e \mu V}{\gamma L^{2}} \tau P
$$

where $\mathrm{e}$ is the electron charge, $\mathrm{A}$ is fraction of the radiation that is absorbed, $\tau$ is the 
carrier lifetime, $\mu$ is the carrier mobility, $P$ is the incident power, $\gamma$ is the energy required to produce electron hole pair (4.3 eV in GaAs, $15 \mathrm{eV}$ in diamond), and $\mathrm{L}$ is the detector electrode separation. The current is proportional to the incident energy flux as well as the carrier lifetime. The fast time response is possible since the current is proportional to the conductivity and NOT the collected charge.

The current flows through the higher conductivity regions of the PCD when illuminated with radiation. The conduction region can be in either the surface or the bulk material depending on the absorption region of the radiation. The mean free path of photons in a diamond is about $1 \mu \mathrm{m}$ for photons $<1 \mathrm{keV}$ in energy and $1 \mathrm{~mm}$ for $10 \mathrm{keV}$ photons. When absorption is strong ( $<2 \mathrm{keV} \mathrm{X}$-rays), light is absorbed close to the surface. For surface conduction, the charge collection distance is given by $d=\mu \tau V_{\text {bias }} / L^{6}$. To form a continuous conducting layer across the face of the PCD, it must be illuminated within a distance, $\mathrm{d}$, of the electrical contacts. For natural diamond $\mathrm{d} \sim 100 \mu \mathrm{m}$

Electrically, a PCD can be modeled as a capacitor, $\mathrm{C}$, and a resistor, $\mathrm{R}$, in parallel that are in series with a resistor $\mathrm{S}$ (see Figure 1). The capacitance is constant and $\mathrm{R}$ is inversely proportional to the power of the illuminating radiation. The resistance $S$ is the fully saturated PCD resistance value. The cable impedance is $Z_{0}$. With this electrical model the current as a function of the dose rate is not linear. Hodson and Canada have derived a relationship to correct the observed signal to higher approximately linear signals $\left[{ }^{7}\right]$.

$$
I_{\text {corr }}=\frac{V_{\text {bias }}}{\left(\frac{V_{\text {bias }}}{I}\right)-S-Z_{0}}
$$

The system impedance, the hard saturation and the bias voltage must be known fairly well.

Diamond is a natural photoconductive detector. GaAs does not work as a PCD until after irradiation. Irradiating a GaAs sample with neutrons damages the lattice and transforms it into a photoconductive material. This damage decreases the carrier lifetime, $\tau$, and makes the PCD faster. The gain of the PCD can be written as $\left[^{8}\right]$ :

$$
G=\frac{\tau \mu V_{\text {bias }}}{L^{2}}
$$


Therefore, the current or sensitivity from a GaAs PCD decreases as the time response increases.

In this paper, three different PCDs are compared. Two are diamond PCDs. The natural diamond detectors are produced by D-RAD Inc. They are $1 \times 3 \times 1 \mathrm{~mm}(\mathrm{WxLxH})$ The other diamond PCD is created through chemical vapor deposition (CVD) and was packaged at LLNL. The diamond was $10 \mathrm{~mm}$ in diameter and $1 \mathrm{~mm}$ thick. On each side of the diamond were coated electrical contacts $8 \mathrm{~mm}$ in diameter Au. Two GaAs PCDs are GaAs-100ps and GaAs-30ps and are from NuTrek. The GaAs PCDs are 1x3x1 mm (WxLxH).

\section{Time Response:}

The characteristics of the diamond and the GaAs PCDs have been investigated using short pulse laser light. The laser system used is a Ti:Sapphire, Chirped Pulse Amplification (CPA) based Class 4 femtosecond laser system. The output of the laser amplifier is $800 \mathrm{~nm}, 150 \mathrm{fs}$ (nominal), $10 \mathrm{~mJ}$ (nominal), at $10 \mathrm{~Hz}$ repetition rate. The output is propagated through a set of non-linear, BBO crystals to generate $4 \omega(200 \mathrm{~nm})$ light pulses. The pulses were used to illuminate and to characterize the PCDs. The spot size of the laser was $6 \mathrm{~mm}$ in diameter and was larger than the area of the PCDs. The recorders used for these tests were a TDS 694C and TDS 6804 with a bandwidth of 3 $\mathrm{GHz}$ and $8 \mathrm{GHz}$, respectively. The recorded response of the detectors depends only on the intrinsic response of the recorder and the detector since the laser pulse is so short. For these tests all the PCDs were biased with $100 \mathrm{~V}$. The PCD responses were recorded at different levels of intensity of the laser. This was achieved through the use of neutral density filters. This data was used to determine the time responses, dynamic range and linearity of the PCDs.

Typical time responses of the four different PCDs to the laser pulse are given in Figure \#2. The CVD detectors had the slowest response of $\tau_{(10-90)} \sim 170 \mathrm{ps}$. The $\tau_{(10-90)}$ reported is the transition time of the signal from $10 \%$ to $90 \%$ of its full range. The Natural Diamond PCDs transition time was on the order of $\sim 100$ ps. The $\tau_{(10-90)}$ of the GaAs PCDs were given by NuTrek to be $30 \mathrm{ps}$ and a 100 ps. The measured response times of the to PCDs are almost identical. The GaAs-100ps and GaAs-30ps transition 
time were $\sim 70 \mathrm{ps}$ and $60 \mathrm{ps}$, respectively. The full width half maximum time, $\tau_{(\mathrm{FWHM})}$, of the recorded pulse of the CVD and the natural diamond PCD were $\sim 500$ ps and $\sim 200$ ps, respectively. They both had fairly long decays. The $\tau_{(\mathrm{FWHM})}$ of the GaAs PCDs was $\sim 100$ ps. It should be noted that the response time of the TDS6804 was close to the measured response time of the GaAs PCDs. The reported time responses are probably an upper limit.

\section{Linearity/Dynamic Range:}

The linearity and dynamic range for each type of PCD were also investigated by varying the laser energy deposited onto the PCD. The energy deposited on the detector under study was varied from 0.001 to $10 \mu \mathrm{J}$ by attenuating the laser with neutral density filters. Figure \#3 shows the plot of peak voltage from the different types of PCDs as a function of deposited energy. Each PCD type had a different sensitivity to the laser light. The Natural Diamond and he GaAs-100ps had the highest sensitivity to the laser light. The GaAs-30ps PCDs were about a factor of 10 less sensitive than the GaAs-100ps ones. This difference is roughly consistent with NuTrek's specifications. The CVD PCDs had the least sensitivity. The natural diamond data is a compilation of 10 PCDs. Two GaAs30ps PCDs were included in the figure. The other data are from single detectors. All the PCDs are fairly linear over at least 2-3 orders of magnitude. This was the limit of the measured range. The GaAs-100ps detector appears to show some non-linearity at about 1 $\mu \mathrm{J}$ of deposited energy.

\section{X-ray Characterizations:}

The Natural Diamond PCDs were irradiated at the Brookhaven synchrotron light source with X-rays between 2 - $6 \mathrm{keV}$. The PCDs were placed behind different Ta apertures which were used to block the X-ray from all surfaces but the PCD active area. Apertures of several sizes were used. One just allowed X-rays onto the active surface of the PCD and was $1 \times 1 \mathrm{~mm}$ square. The other allowed X-rays to hit the electrical contacts of the PCDs. The PCD was also scanned systematically into and out of the beam. The PCD response was substantially different depending on where the X-rays hit the detector. 
The response changed dramatically whether or not the PCD active area was illuminated within a diffusion length of the electrical contact. If the entire PCD area was not illuminated, the increased conductivity region did not connect both electrical contacts and the current recorded was less. Also, the PCD response increased dramatically if the electrical contacts and solder joints were illuminated with X-rays. X-rays hitting the solder joints caused the emission of photo-electrons similar to those created in an XRD. $\mathrm{Sn}$ and $\mathrm{Pb}$ absorption edges in the solder were determined to be the contributors to the photo-electron emission.

\section{Acknowledgments:}

This work was done under the auspices of the U.S. Department of Energy by Lawrence Livermore National Laboratory under Contract DE-AC52-07NA27344. This work was done by National Security Technologies, LLC, under Contract No.

DE-AC52-06NA25946 with the U.S. Department of Energy. 


\section{Figure Captions:}

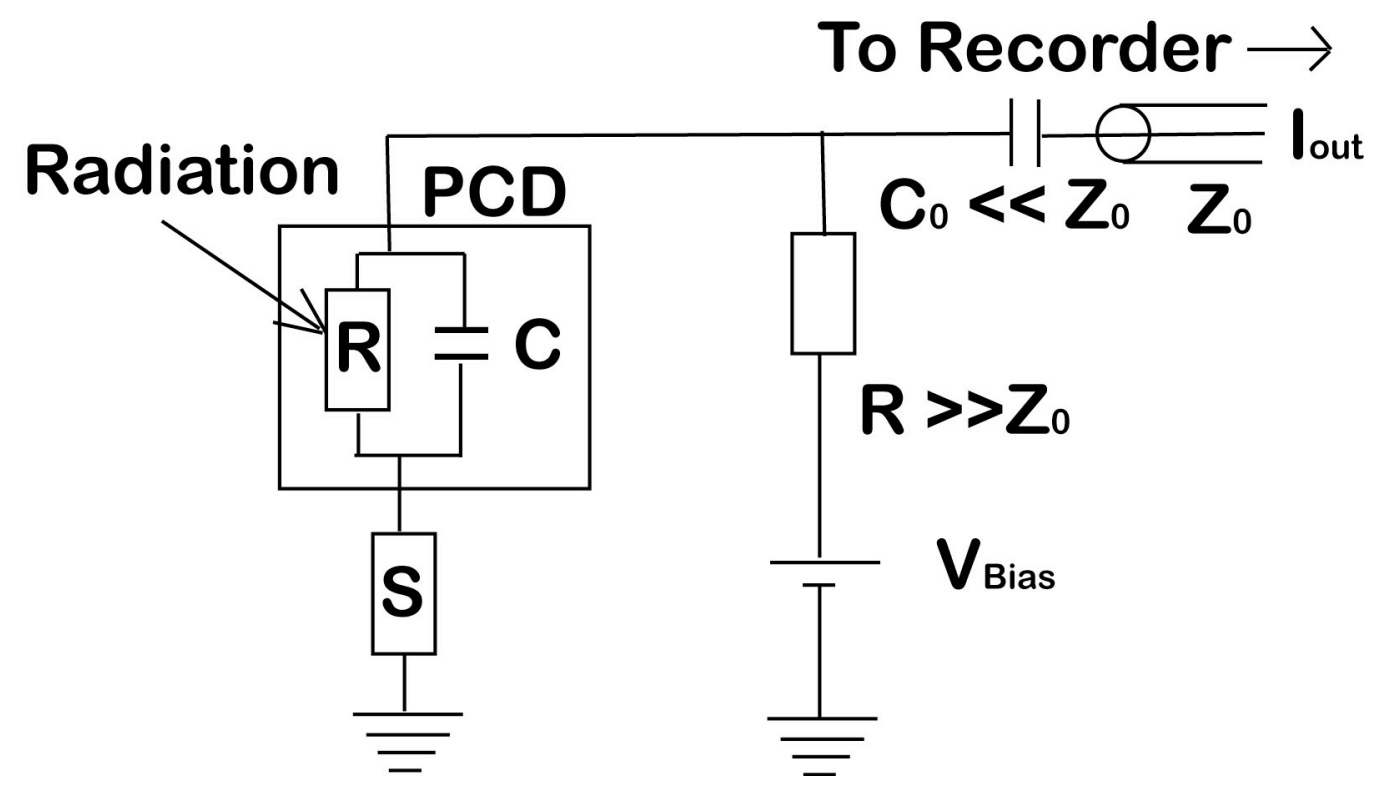

Figure 1: Electrical schematic of the PCD. 


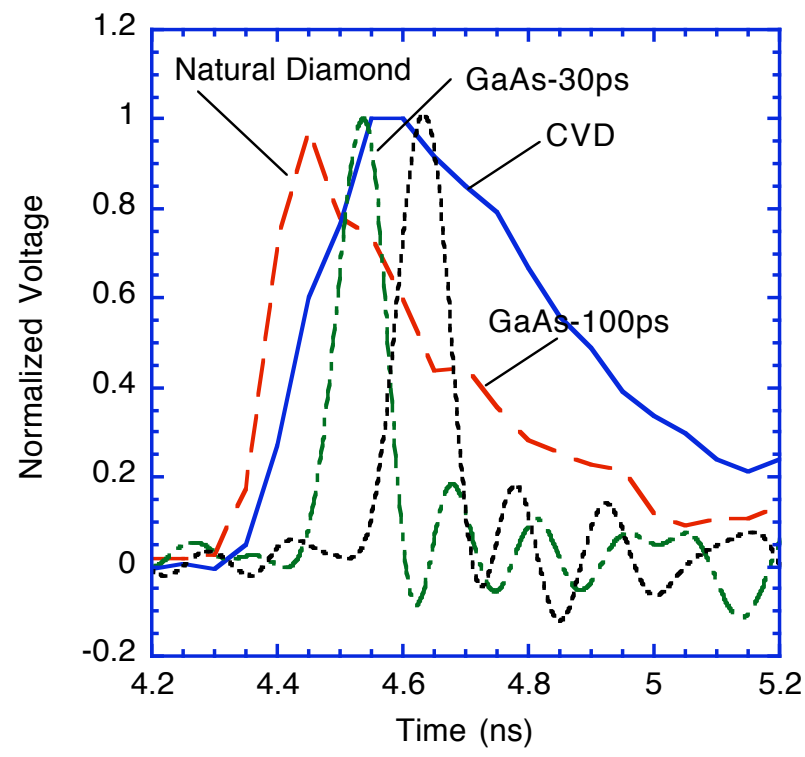

Fig. 2: Time response of several different PCDs when exposed to $200 \mathrm{~nm}$ laser light. 


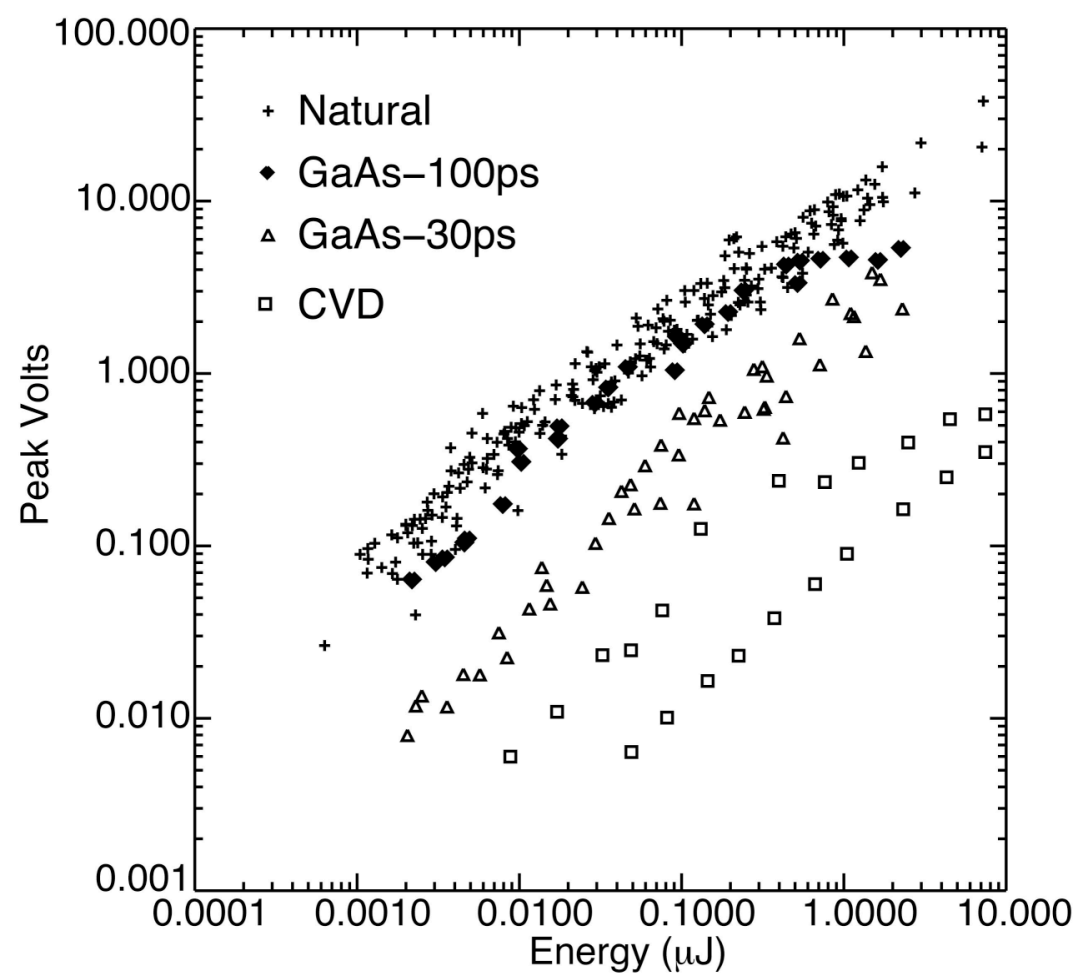

Fig. 3: Linearity and sensitivity of several different PCDs when exposed to $200 \mathrm{~nm}$ laser light. 


\section{References:}

${ }^{1}$ D. E. Hinkel, M. B. Schneider, B. K. Young, et al, Phys. Rev. Lett. 96, 195001 (2006)

${ }^{2}$ M. Derzon, et al., Review of Scientific Instruments, Vol. 70, 1, 566 (1999).

${ }^{3}$ G.A. Chandler, et al., Rev. Scien. Instr. 70, p 561 (1999).

${ }^{4}$ J.E. Rauch, et al., IEEE Pulsed Power, 2005.

${ }^{5}$ G. C. Idzorek and R. J. Bartlett, Proc. SPIE 3114, 3491997

${ }^{6}$ L.S. Pan et al., J. Appl. Phys. 74 (2), 1086 (1993)

${ }^{7}$ E. Hodson and J. Canada, "GaAs Photo-Conductive Detector Behavior in Band Limited Systems with Greater than Zero Impedance", 1992 at EG\&G Energy Measurement Santa Barbara, CA.

${ }^{8}$ H.H. Ziegler, Nucl. Instr. and Meth. in Phys. Res. A, 380, pp 220-222 (1996) 